\title{
Correction to: Loss of Neuropilin2a/b or Sema3fa alters olfactory sensory axon dynamics and protoglomerular targeting
}

\author{
Ryan P. Cheng, Puneet Dang, Alemji A. Taku, Yoon Ji Moon, Vi Pham, Xiaohe Sun, Ethan Zhao and \\ Jonathan A. Raper
}

\section{Correction to: Neural Dev 17, 1 (2022)}

https://doi.org/10.1186/s13064-021-00157-x

Following publication of the original article [1], the last two figures in the paper were misordered during the publication process and references to one of the figures were omitted. The figure entitled "Both nrp2a and nrp2b act in the same pathway with sema3fa" formerly appeared as Fig. 6 and now appears correctly as Fig. 5. The figure entitled "Misprojecting growth cones fail to retract in nrp2a and in sema3fa mutants" formerly appeared as Fig. 5 and now appears as Fig. 6 . The correct figures are given below. Text referring to the corrected Fig. 5 was amended to: "Neither the number of misprojections nor the pattern of misprojections were significantly different between sema3fa-l- and nrp2a-/-;sema3fa-l- animals (Fig. 5A,B). Similarly, the number of misprojections and the pattern of misprojections were not significantly different between sema3fa-/ - and nrp2b-/-;sema3fa-/animals (Fig. 5C,D). Also, the following typographical errors, TRCP2 and TPRC 2 on page 14 and TRCP2 on figure 6 caption were amended to TRPC2. This version of the paper now reflects these corrections to the original.

The original article [1] has been corrected.
Published online: 05 March 2022

\footnotetext{
Reference

1. Cheng RP, Dang P, Taku AA, et al. Loss of Neuropilin2a/b or Sema3fa alters olfactory sensory axon dynamics and protoglomerular targeting. Neural Dev. 2022;17:1. https://doi.org/10.1186/s13064-021-00157-x.
} to the material. If material is not included in the article's Creative Commons licence and your intended use is not permitted by statutory regulation or exceeds the permitted use, you will need to obtain permission directly from the copyright holder. To view a copy of this licence, visit http://creativecommons.org/licenses/by/4.0/. The Creative Commons Public Domain Dedication waiver (http://creativeco mmons.org/publicdomain/zero/1.0/) applies to the data made available in this article, unless otherwise stated in a credit line to the data. 

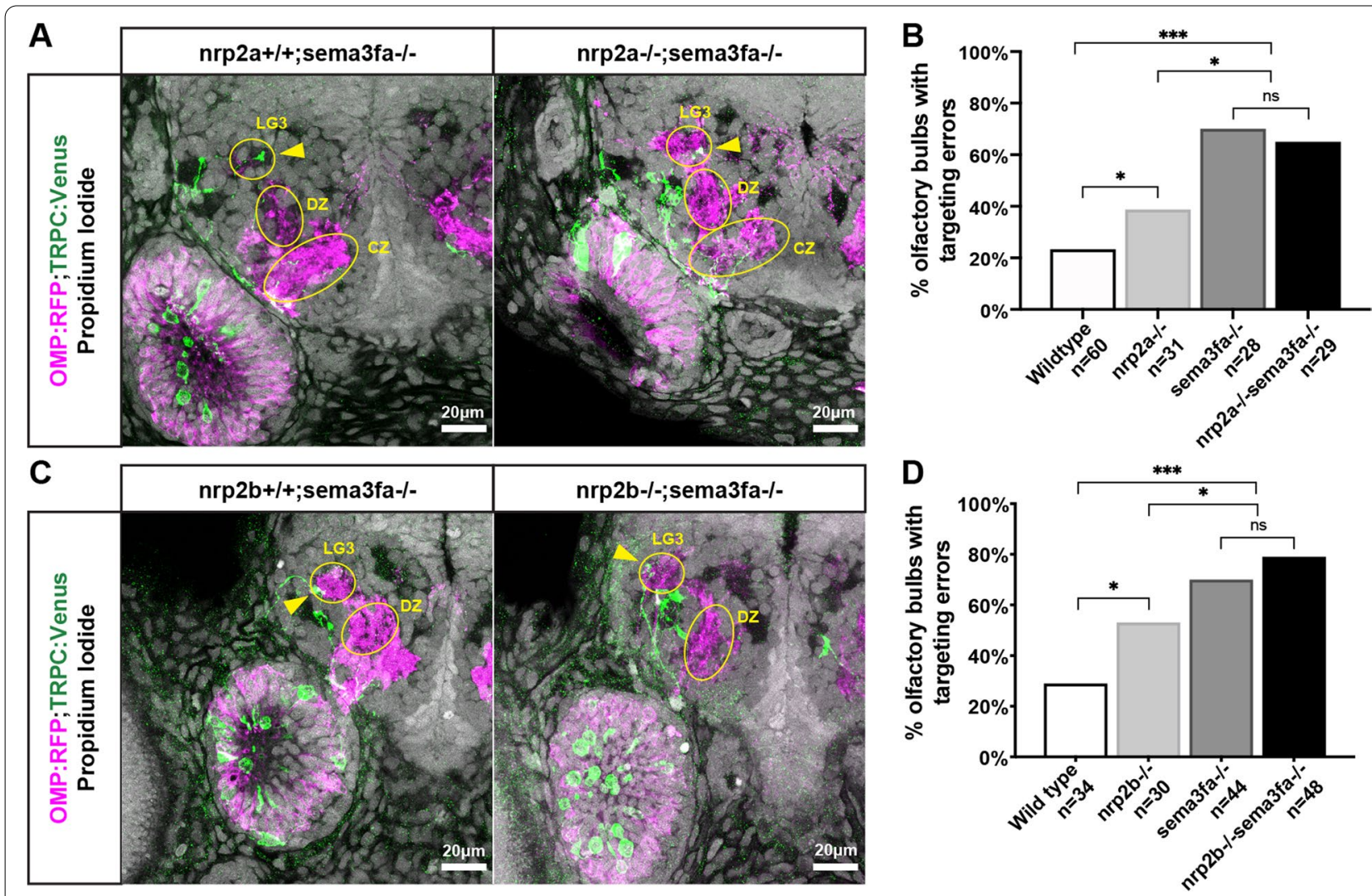

Fig. 5 Both nrp2a and nrp2b act in the same pathway with sema3fa. A Representative confocal sections of sema3fa mutant and nrp2a;sema3fa double mutant siblings. Yellow arrows indicate misprojecting axons. B The misprojection phenotype in nrp2a;sema3fa double mutants is not significantly different from sema3fa mutant siblings. C Representative confocal sections of sema3fa mutant and nrp2b;sema3fa double mutant siblings. Yellow arrows indicate misprojecting axons. D The misprojection phenotype in nrp2b;sema3fa double mutants is not significantly different from sema3fa mutant siblings. Model of average TRPC2-class axon locations in sema3fa heterozygotes and mutants during live imaging sequence. TRPC2-class axons are shown in green and magenta, and OMP-class axons in grey. The three TRCP2 surfaces encompass axon location probabilities, from most transparent to most opaque, of 5.6, 18.7, and 31.8\%. Yellow dotted line represents the edge of the dorsal-medial OB region

(See figure on next page.)

Fig. 6 Misprojecting growth cones fail to retract in nrp2a and in sema3fa mutants. A Live imaging sequences of nrp2a heterozygote and mutant siblings, showing misprojecting axons occupying the dorsal-medial OB. The yellow dotted lines indicate the dorsal boundary of the developing $\mathrm{DZ}$ and $\mathrm{CZ}$ protoglomeruli and denote the edge of the dorsal-medial OB region. Yellow arrows indicate misprojecting axons. B The cumulative time that the dorsal-medial OB is occupied by TRPC2-class OSNs is greater in nrp2a mutants as compared to nrp2a heterozygous siblings. C The maximum distance TRPC2-class axons project into the dorsal-medial OB is greater in nrp2a mutants as compared to heterozygotes. $\mathbf{D}$ Live imaging sequences of sema3fa heterozygote and mutant siblings, showing misprojecting axons occupying the dorsal-medial OB. EThe cumulative time that the dorsal-medial OB is occupied by TRPC2-class OSNs is greater in sema3fa mutants as compared to heterozygous siblings. F The maximum distance TRPC2-class axons project into the dorsal-medial OB is greater in sema3fa mutants as compared to heterozygotes. G Model of average TRPC2-class axon locations in sema3fa heterozygotes and mutants during live imaging sequence. TRPC2-class axons are shown in green and magenta, and OMP-class axons in grey. The three TRPC2 surfaces encompass axon location probabilities, from most transparent to most opaque, of 5.6, 18.7, and 31.8\%. Yellow dotted line represents the edge of the dorsal-medial OB region 


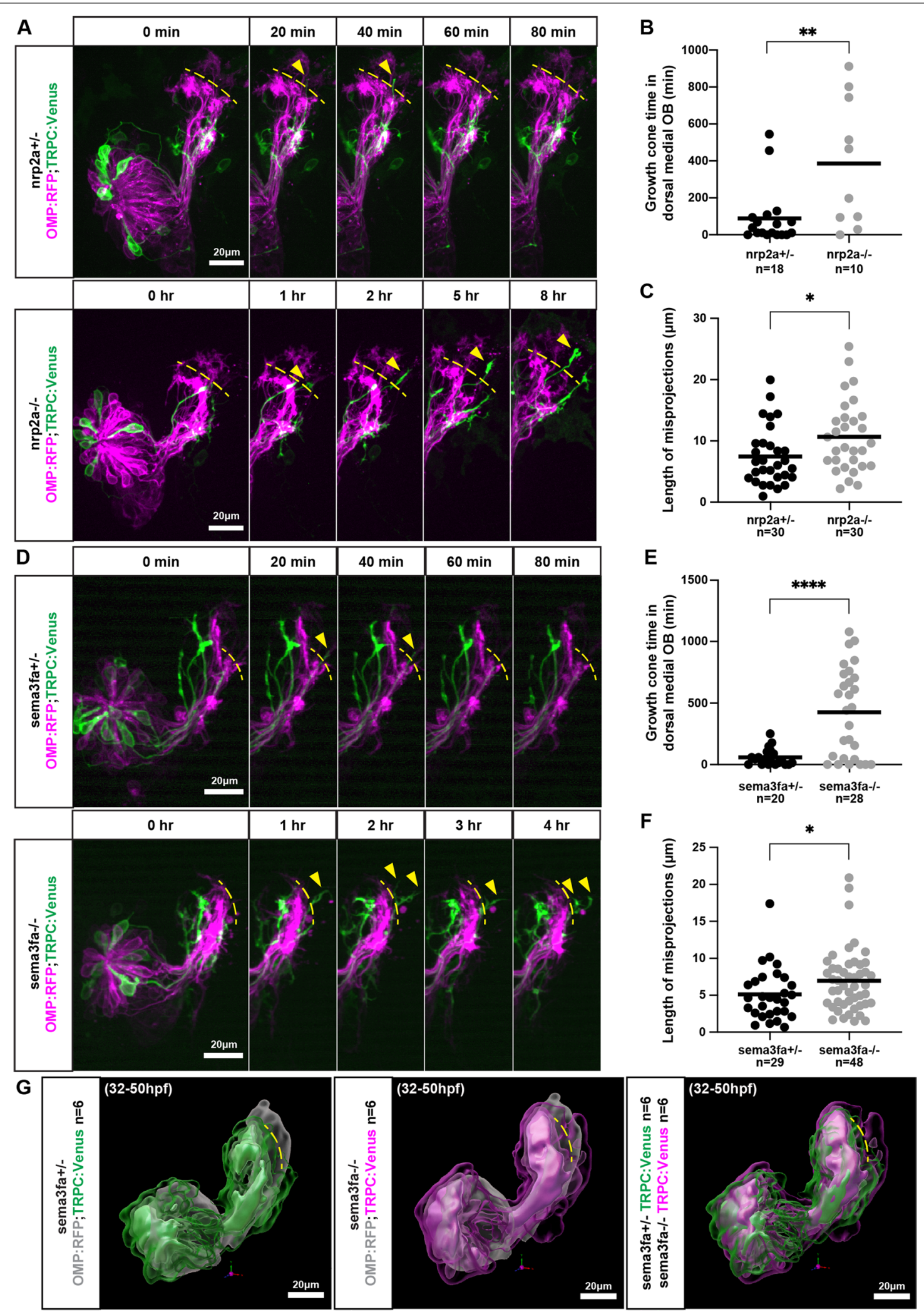

Fig. 6 (See legend on previous page.) 